\title{
Real-World Experience on Omalizumab Treatment for Patients with Normocomplementemic Urticarial Vasculitis
}

\author{
Taoming Liu \\ Juan Bai \\ Shuni Ying \\ Sheng Li (D) \\ Yunlei Pan \\ Deren Fang \\ Jianjun Qiao (iD \\ Hong Fang (iD
}

Department of Dermatology, The First Affiliated Hospital, Zhejiang University School of Medicine, Hangzhou, People's Republic of China
Correspondence: Jianjun Qiao; Hong Fang Department of Dermatology, The First Affiliated Hospital, Zhejiang University School of Medicine, No.79 Qingchun Road, Hangzhou, People's Republic of China

Tel/Fax +86-57I-87236706

Email qiaojianjun@zju.edu.cn;

fanghongzy@zju.edu.cn
Background: Urticarial vasculitis is a small vessel vasculitis characterized by long-lasting wheals. It was suggested omalizumab is well tolerated and effective in patients with hypocomplementaemic urticarial vasculitis.

Objective: To evaluate the clinical response and safety of omalizumab for treating patients with normocomplementaemic urticarial vasculitis (NUV) in real-world setting.

Methods: We collected data from a single-center. This study included patients with NUV who was received omalizumab therapy. During a 24-week study period, the clinical efficacy was evaluated by patient's self-assessment instrument urticarial vasculitis activity score and Dermatology Life Quality Index.

Results: Five patients with NUV were enrolled. Three patients received 6 doses of 150 or $300 \mathrm{mg}$ omalizumab subcutaneously every 4 weeks. At 24 -week follow-up, it was revealed improvement of clinical manifestations and reduction of urticarial vasculitis activity score and Dermatology Life Quality Index. At 24-week visit, mild wheals recurred in one patient who was only administrated with omalizumab for 4 times. One patient did not response to omalizumab therapy. No adverse events were recorded in the 5 patients.

Conclusion: Omalizumab may be a potential choice in the treatment of patients with NUV in the real-world life.

Keywords: normocomplementemic urticarial vasculitis, omalizumab, real-world, targeted therapy, $\operatorname{IgE}$

\section{Introduction}

Urticarial vasculitis (UV) is considered as a chronic and idiopathic inflammatory skin disease. ${ }^{1}$ According to the serum complement level, UV can be classified as hypocomplementaemic and normocomplementaemic urticarial vasculitis (NUV). ${ }^{2} \mathrm{NUV}$ is always represented by cutaneous symptoms, including long-lasting wheals, residual skin pigmentation, and itch. ${ }^{3}$ Biopsy findings of UV is characterized by predominantly lymphocytic infiltrate accompanied by eosinophils and red blood cell extravasation. ${ }^{4}$

Various traditional therapies have been tried to administrate in patients with UV, including glucocorticoids, hydroxychloroquine, thalidomide, and oral antihistamines, but these treatments showed unsatisfied responses or dose-dependent adverse effects. ${ }^{3}$

Experience with omalizumab, an anti-IgE monoclonal antibody, has been used for treating hypocomplementaemic urticarial vasculitis. ${ }^{5-8}$ In three case reports, NUV patients revealed remarkable response to omalizumab. ${ }^{9-11}$ A prospective, open-label proof-of-concept study in a single center show that patients with NUV can benefit from 
omalizumab treatment during 12-week follow-up. It was demonstrated that 4 patients $(17.4 \%)$ achieved complete response and 13 patients (56.5\%) got partial response. ${ }^{12}$ In fact, real-world evidence could add data that cannot obtain from clinical trials, including treatment course, administration, long-term safety and effectiveness in routine clinical therapies. ${ }^{13}$ It is not yet clear of the efficacy of omalizumab for treating patients with NUV. Our current real-world study provides evidence of the efficacy and safety of omalizumab for treating NUV patients.

\section{Materials and Methods}

We collected data of omalizumab treatment for patients with NUV in clinical real-world from a single-center. Inclusion criteria included patients diagnosed with NUV and received omalizumab treatment in The First Affiliated Hospital, Zhejiang University School of Medicine, during October 1, 2019-January 20, 2021. This study was conducted according to the Declaration of Helsinki. This study protocol was approved by the Ethics Committee of The First Affiliated Hospital, Zhejiang University School of Medicine (Approved number: IIT-2021-088). All the patients have given the written informed consent to this case series with all details displayed.

All the patients $(\mathrm{n}=5)$ administrated subcutaneously an offlabel omalizumab treatment at doses of either $150 \mathrm{mg}$ or $300 \mathrm{mg}$ every 4 weeks. We collected data from medical records, including clinical symptoms, laboratory results, the patients' general condition, photographs, previous treatments before omalizumab, and adverse effects during therapy period.

The clinical response of patients treated with omalizumab was evaluated by urticarial vasculitis activity score (UVAS) and the Dermatology Life Quality Index (DLQI) at fixed time points (week $0,4,8,12,16,20$, and 24). The UVAS are composed of 5 key UV symptom values, including wheals, pruritus, residual skin pigmentation, arthralgia, and general symptoms (fatigue, exhaustion, chills, and fever). ${ }^{14}$ The score ranges from 0 to 10 , with 0 indicating none and 10 indicating very severe. The value of total UVAS was determined as the mean of 5 subscale values. ${ }^{14}$ The patients' quality of life (QoL) was assessed by DLQI (score range of 0 to 30, where lower scores indicating better QoL). ${ }^{15,16}$

\section{Results}

Five patients with NUV treated with omalizumab (4 female; 1 male) were included in our study. Tables 1 and 2 summarize the clinical characteristics, laboratory

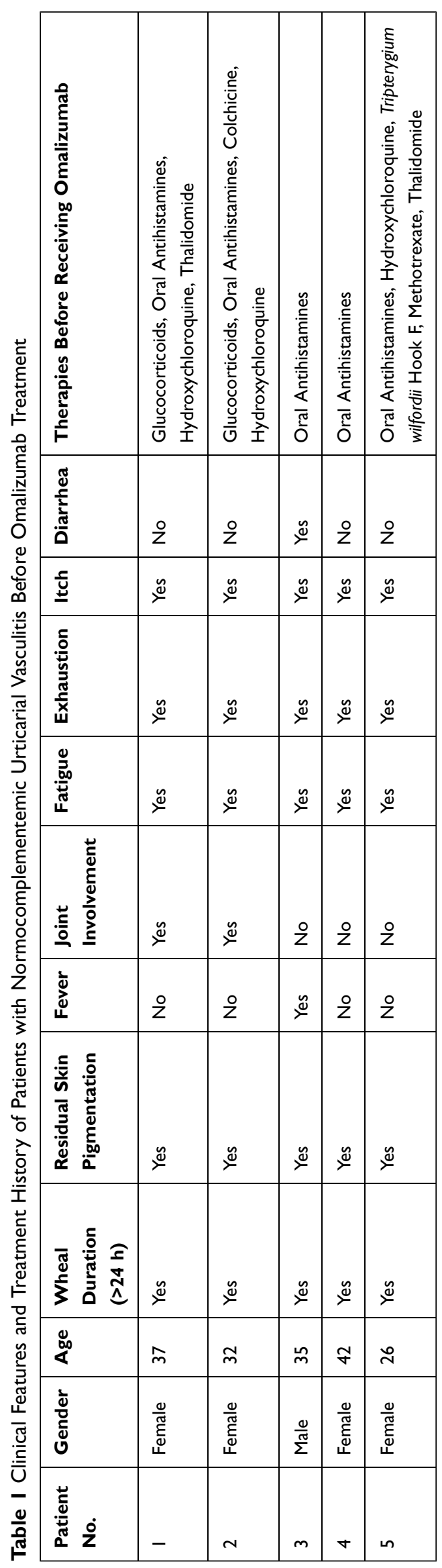


Table 2 Laboratory Features and Omalizumab Treatment Protocol of Patients with Normocomplementemic Urticarial Vasculitis

\begin{tabular}{|c|c|c|c|c|c|c|c|c|}
\hline $\begin{array}{l}\text { Patient } \\
\text { No. }\end{array}$ & $\begin{array}{l}\text { Serum C4 } \\
(\mathrm{mg} / \mathrm{dl})\end{array}$ & $\begin{array}{l}\text { WBC Count } \\
\left(10^{9} / L\right)\end{array}$ & $\begin{array}{l}\text { Neutrophil } \\
\text { Count }\left(10^{9} / \mathrm{L}\right)\end{array}$ & $\begin{array}{l}\mathrm{IL-6} \\
(\mathrm{pg} / \mathrm{mL})\end{array}$ & $\begin{array}{l}\text { ESR } \\
(\mathrm{mm} / \mathrm{h})\end{array}$ & $\begin{array}{l}\text { CRP } \\
\text { (mg/L) }\end{array}$ & $\begin{array}{l}\text { Serum Total IgE } \\
\text { (KU/L) }\end{array}$ & $\begin{array}{l}\text { Dosage/Course of } \\
\text { Omalizumab Treatment }\end{array}$ \\
\hline 1 & 23 & 8 & 5.9 & 3.59 & 6 & 3.7 & 19.4 & $150 \mathrm{mg}$ Every 4 weeks $/ 6$ \\
\hline 2 & 29 & 10 & 7.7 & NA & 4 & 13.8 & 396 & 150mg Every 4 weeks $/ 6$ \\
\hline 3 & 21 & 8.7 & 5.9 & NA & 1 & 3.1 & 542 & $300 \mathrm{mg}$ Every 4 weeks $/ 6$ \\
\hline 4 & 23 & 6.2 & 3.4 & 3.11 & 5 & $<3$ & 132 & 150mg Every 4 weeks $/ 4$ \\
\hline 5 & 35 & 9.1 & 6.9 & 8.1 & 12 & 20.3 & 214 & $150 \mathrm{mg}$ Every 4 weeks $/ 3$ \\
\hline
\end{tabular}

Abbreviations: C4, complement C4; WBC, leukocyte; IL-6, interleukin-6; ESR, erythrocyte sedimentation rate; CRP, C reactive protein; Total lgE, immunoglobulin E.

features, and previous therapies for NUV recorded at the start of the treatment.

As shown in Table 1, patients presented various extracutaneous symptoms, including fatigue $(n=5)$, exhaustion $(n=5)$, arthralgia $(n=2)$, fever $(n=1)$, and diarrhea $(n=1)$ before treatment with omalizumab, respectively. Four patients represented elevated serum total IgE levels, that is $132-542 \mathrm{KU} / \mathrm{L}$ at baseline (normal range $<100.0 \mathrm{KU} / \mathrm{L}$ ). Therapies before starting omalizumab treatment for each patient were listed in Table 1. In our case series, all patients did not response to oral H1antihistamines. In addition, patient 3, 4, and 5 decline to receive oral corticosteroids and immunosuppressants therapy, due to afraid of potentially serious adverse effects.

During the 24-week study period, treatment with omalizumab for NUV achieved significant improvement of disease activity and QoL as measured by UVAS and DLQI. The changes of UVAS and DLQI of each patient are described in Figure 1. Three patients (patient 1, 2, and 3) received omalizumab treatment for 6 times, dose of $150 \mathrm{mg}$ (patient 1 and 2) or $300 \mathrm{mg}$ (patient 3) every 4 weeks. Compared with the baseline, the values of UVAS and DLQI notably decreased at the 12-week visit, and both of them persistently reduced at the 24-week assessment.

At 16-week visit, patient 4 discontinued the treatment with omalizumab as she was satisfied with the overall therapeutic benefit. Although mild relapse with less wheals was reported at 24-week follow-up, the patient declined further omalizumab treatment because she can resistant to her mild symptoms.

At the 12-week visit, patient 5 decided to terminate treatment with omalizumab due to new lesions. There were no adverse events observed during omalizumab treatment.

\section{Discussion}

In this case series, we report the efficacy of anti-IgE omalizumab therapy in NUV. The notable reductions of disease activity and QoL were observed. Consistently high effectiveness was also demonstrated throughout the entire study period.

To our knowledge, the role of $\mathrm{IgE}$ in the pathogenesis of NUV has not been elucidated. Omalizumab, a humanized anti-IgE $\mathrm{mAb}$, is effective for the patients with chronic spontaneous urticaria and asthma. ${ }^{17}$ Based on high level of IgE in serum and the similarity of cutaneous lesions to chronic spontaneous urticaria, omalizumab may be effective in patients with UV through its reduction of IgE level. ${ }^{18}$ The patients with high serum IgE levels appear to respond to omalizumab better in the treatment of NUV. Moreover, case reports from other centers have suggested that omalizumab treatment is effective in patients with UV. ${ }^{18-20}$ The finding may prompt scientists to reveal the role of $\mathrm{IgE}$ in the pathogenesis of NUV.

The disease activity and QoL of our patients (patient 1, 2 , and 3) progressively and steadily decreased over the entire study period. These findings are consistent with previous case reports, highlighting the significant efficacy of omalizumab for the treatment of NUV.

Nevertheless, slight wheals reoccurred in patient 4 after two months from discontinuation of omalizumab. It is possible that the result was influenced by the less treatment courses (4 times) and dosage (150 mg). $150 \mathrm{mg}$ dosage may be relevant in improving severity, that is sufficient administration might be recommended at the start of treatment with omalizumab. ${ }^{19}$ Noteworthy, patient 5 showed no response to and interrupted omalizumab treatment during 12 -week therapies. It is probably that the elevated serum IL-6 may also be interpreted as the reason for ineffective of treatment with omalizumab. IL- $1 \beta$ is thought to draw a contribution to the pathogenesis of $\mathrm{UV}$, through promoting vessel permeability and neutrophils chemotaxis. $^{21}$ IL-1 $\beta$ antagonist was confirmed to have a potential efficacy in patients with $\mathrm{UV}^{14,22} \mathrm{We}$ hypothesize that inhibiting IL-1 $\beta$ may lead to 

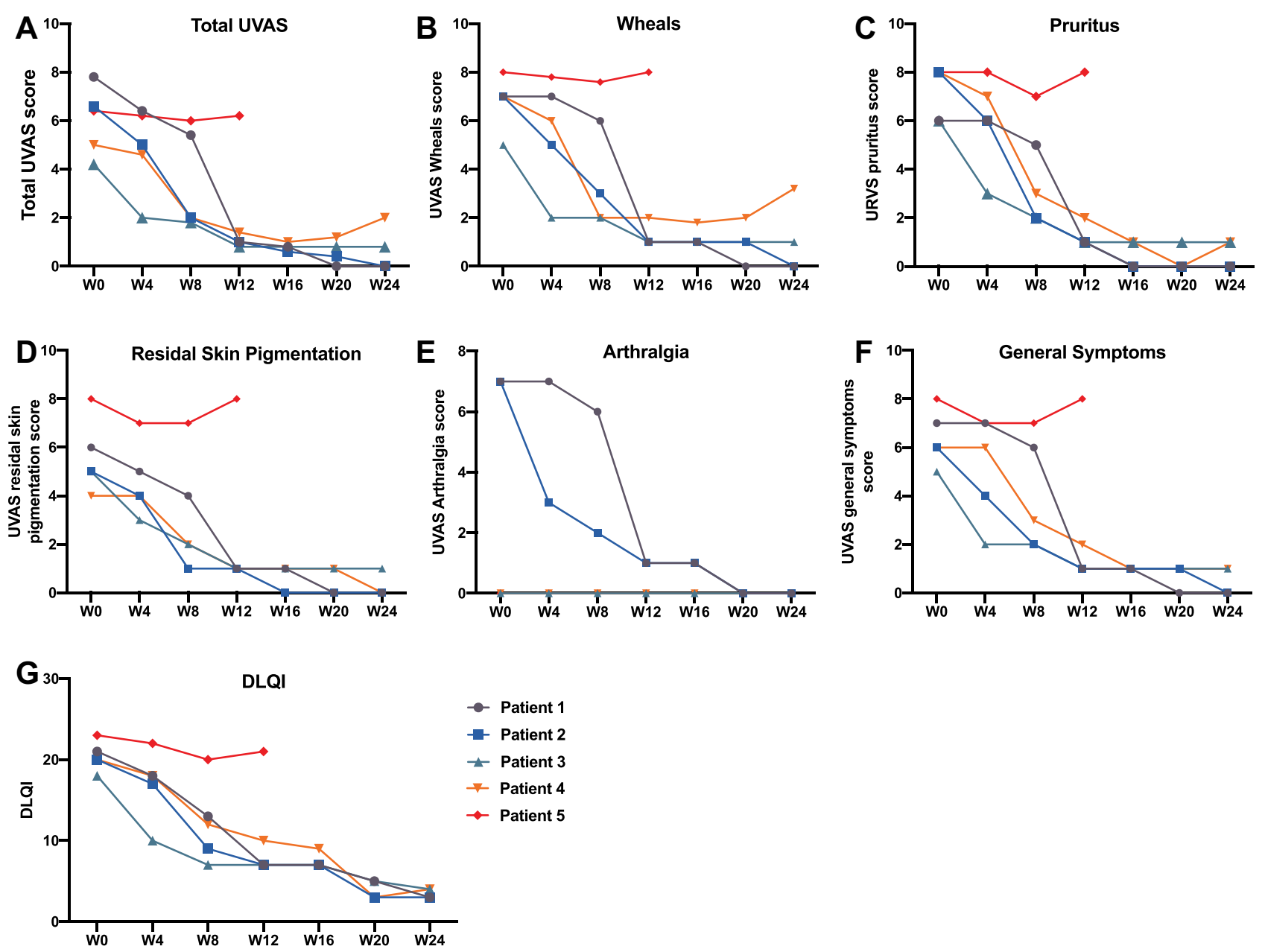

Figure I Disease activity and quality of life of patients with normocomplementemic urticarial vasculitis during the 24-week study period. (A) Total UVAS score, (B) UVAS wheals score, (C) UVAS pruritus score, (D) UVAS residual skin pigmentation score, (E) UVAS arthralgia score, (F) UVAS general symptoms score, and (G) DLQI. Abbreviations: UVAS, urticarial vasculitis activity score; DLQI, Dermatology Life Quality Index.

a promising efficacy of the patients with NUV who was partially responsive to omalizumab.

It has certain limitations in our present study, including retrospective nature, small number of patients, and lack of the evaluation of efficacy with different treatment dosage and course of omalizumab.

\section{Conclusion}

In summary, our real-world data further support omalizumab may be a potential and well tolerated choice for treating UNV. It is necessary to design a prospective study to evaluate the efficacy and safety of omalizumab for the patients with NUV with large patient number and long-term assessment in multiple centers. In addition, further studies are required to address the following questions, including various dosage of omalizumab and the problem of relapse after discontinuing treatment.

\section{Acknowledgments}

This work was supported by the National Natural Science Foundation of China (81972931 and 81673045 to HF), the Medical and Health Science and Technology Project of Health Commission of Zhejiang Province (2020KY558 to JQ, 2014KYA061 to DF).

\section{Disclosure}

The authors declare no conflicts of interest related to this work.

\section{References}

1. Venzor J, Lee WL, Huston DP. Urticarial vasculitis. Clin Rev Allergy Immunol. 2002;23(2):201-216. doi:10.1385/criai:23:2:201

2. Tosoni C, Lodi-Rizzini F, Cinquini M, et al. A reassessment of diagnostic criteria and treatment of idiopathic urticarial vasculitis: a retrospective study of 47 patients. Clin Exp Dermatol. 2009;34 (2):166-170. doi:10.1111/j.1365-2230.2008.02891.x 
3. Kolkhir P, Grakhova M, Bonnekoh H, Krause K, Maurer M. Treatment of urticarial vasculitis: a systematic review. J Allergy Clin Immunol. 2019;143(2):458-466. doi:10.1016/j.jaci.2018.09.007

4. Lee JS, Loh TH, Seow SC, Tan SH. Prolonged urticaria with purpura: the spectrum of clinical and histopathologic features in a prospective series of 22 patients exhibiting the clinical features of urticarial vasculitis. J Am Acad Dermatol. 2007;56(6):994-1005. doi:10.1016/ j.jaad.2006.10.962

5. Navarro-Navarro I, Jiménez-Gallo D, Villegas-Romero I, LinaresBarrios M. Use of omalizumab in the treatment of hypocomplementemic urticarial vasculitis. Dermatol Ther. 2020;33(2):e13237. doi:10.1111/dth. 13237

6. Pérez Codesido S, Rosado Ingelmo A, Gómez de la Fuente E, García García E, Privitera Torres M, Tejedor Alonso MA. Successful treatment of hypocomplementemic urticarial vasculitis with omalizumab: a case report. J Investig Allergol Clin Immunol. 2020;30(3):211-212. doi:10.18176/jiaci.0486

7. Aurich S, Simon JC, Treudler R. Omalizumab does not improve skin lesions in a patient with hypocomplementemic urticarial vasculitis syndrome. J Eur Acad Dermatol Venereol. 2017;31(9):e395-e397. doi:10.1111/jdv.14202

8. Nucera E, Basta F, Buonomo A, et al. A case of hypocomplementemic urticarial vasculitis syndrome successfully treated with omalizumab. J Investig Allergol Clin Immunol. 2017;27 (6):382-384. doi:10.18176/jiaci.0191

9. Rattananukrom T, Svetvilas P, Chanprapaph K. Successful treatment of normocomplementemic urticarial vasculitis with omalizumab: a report of three cases and literature review. Asian Pac J Allergy Immunol. 2020;38(4):286-289. doi:10.12932/ap-050918-0402

10. Ito T, Maeda T, Egusa C, et al. Effectiveness of omalizumab in two cases of normocomplementemic urticarial vasculitis due to perivascular mast cell infiltration. Allergol Int. 2020. doi:10.1016/j. alit.2020.09.012

11. de Brito M, Huebner G, Murrell D, Bullpitt P, Hartmann K. Normocomplementaemic urticarial vasculitis: effective treatment with omalizumab. Clin Transl Allergy. 2018;8:37. doi:10.1186/ s13601-018-0222-y

12. Chen YD, Krause K, Tu P, Zhao ZT, Maurer M. Response of omalizumab in normocomplementemic urticarial vasculitis. $J$ Allergy Clin Immunol Pract. 2020;8(6):2114-2117.e2. doi:10.1016/j.jaip.2020.02.024
13. Raphael MJ, Gyawali B, Booth CM. Real-world evidence and regulatory drug approval. Nat Rev Clin Oncol. 2020;17(5):271-272. doi:10.1038/s41571-020-0345-7

14. Krause K, Mahamed A, Weller K, Metz M, Zuberbier T, Maurer M. Efficacy and safety of canakinumab in urticarial vasculitis: an open-label study. J Allergy Clin Immunol. 2013;132(3):751-754.e5. doi:10.1016/j.jaci.2013.04.008

15. Lennox RD, Leahy MJ. Validation of the Dermatology Life Quality Index as an outcome measure for urticaria-related quality of life. Ann Allergy Asthma Immunol. 2004;93(2):142-146. doi:10.1016/s10811206(10)61466-4

16. Młynek A, Zalewska-Janowska A, Martus P, Staubach P, Zuberbier T, Maurer M. How to assess disease activity in patients with chronic urticaria? Allergy. 2008;63(6):777-780. doi:10.1111/j.13989995.2008.01726.x

17. Maurer M, Rosén K, Hsieh HJ, et al. Omalizumab for the treatment of chronic idiopathic or spontaneous urticaria. $N$ Engl J Med. 2013;368(10):924-935. doi:10.1056/NEJMoa1215372

18. Fueyo-Casado A, Campos-Muñoz L, González-Guerra E, PedrazMuñoz J, Cortés-Toro JA, López-Bran E. Effectiveness of omalizumab in a case of urticarial vasculitis. Clin Exp Dermatol. 2017;42 (4):403-405. doi:10.1111/ced.13076

19. Cherrez-Ojeda I, Vanegas E, Felix M, Mata VL, Cherrez A. Patientreported outcomes in urticarial vasculitis treated with omalizumab: case report. BMC Dermatol. 2018;18(1):8. doi:10.1186/s12895-0180077-x

20. Ghazanfar MN, Thomsen SF. Omalizumab for urticarial vasculitis: case report and review of the literature. Case Rep Dermatol Med. 2015;2015:576893. doi:10.1155/2015/576893

21. Davis MDP, van der Hilst JCH. Mimickers of urticaria: urticarial vasculitis and autoinflammatory diseases. J Allergy Clin Immunol Pract. 2018;6(4):1162-1170. doi:10.1016/j.jaip.2018.05.006

22. Bettuzzi T, Deroux A, Jachiet M, et al. Dramatic but suspensive effect of interleukin-1 inhibitors for persistent urticarial vasculitis: a French multicentre retrospective study. Ann Rheum Dis. 2019;78 (10):1446-1448. doi:10.1136/annrheumdis-2019-215605
Journal of Asthma and Allergy

\section{Publish your work in this journal}

The Journal of Asthma and Allergy is an international, peer-reviewed open-access journal publishing original research, reports, editorials and commentaries on the following topics: Asthma; Pulmonary physiology; Asthma related clinical health; Clinical immunology and the immunological basis of disease; Pharmacological interventions and new therapies. The manuscript management system is completely online and includes a very quick and fair peer-review system, which is all easy to use. Visit http://www.dovepress.com/testimonials.php to read real quotes from published authors. 